\title{
Bionic Biphasic Composite Scaffold with Osteochondrogenic Factors for Regeneration of Full-thickness Osteochondral Defect
}

\section{Yanbo Zhang}

Jilin University No 3 Hospital: China-Japan Union Hospital of Jilin University

\section{Yu Han}

Jilin University Second Hospital

\section{Yachen Peng}

Jilin University No 3 Hospital: China-Japan Union Hospital of Jilin University

Jie Lei

Jilin University

Fei Chang ( $\nabla$ ccfei_cn@hotmail.com )

Jilin University Second Hospital

\section{Research}

Keywords: Bionic biphasic composite scaffold, Osteochondrogenic factor, Regeneration, Full-thickness osteochondral defect

Posted Date: August 6th, 2021

DOl: https://doi.org/10.21203/rs.3.rs-750461/v1

License: (c) (1) This work is licensed under a Creative Commons Attribution 4.0 International License.

Read Full License

Version of Record: A version of this preprint was published at Biomaterials Science on January 1st, 2022. See the published version at https://doi.org/10.1039/D2BM00103A. 


\section{Abstract}

Background: Full-thickness osteochondral defects lack the capability to self-repair owing to their complicated hierarchical structure. At present, clinical treatments including microfracture etc. have shown some efficacy; however, the newborn tissue exhibits some drawbacks, such as fibrocartilage formation and insufficient mechanical properties, eventually leading to osteoarthritis. Moreover, cartilage and bone tissues have different biological characteristics. Therefore, the simultaneous repair of full-thickness osteochondral defects is highly challenging.

Methods: To achieve optimal repair, we constructed a bionic biphasic composite scaffold with ondemand osteochondrogenic factors. Kartogenin and extractive mesenchymal stem cells were introduced into a mPEG-block-poly(L-valine) thermogel set as the upper layer to accelerate cartilage repair. A poly(lactide-co-glycolide)/hydroxyapatite porous scaffold was immobilized with bone morphogenetic protein-2 as the bottom layer to facilitate subchondral repair. The intermediate interface, being a combination of the two layers was expected to reconstruct the calcification zone.

Results: Gross evaluation, micro-computed tomography, and magnetic resonance imaging indicated that at 6 months after implantation, the scaffold encapsulating mesenchymal stem cells and osteochondrogenic factors had a better effect compared with other groups. More importantly, the composition of the new cartilage and bone was confirmed by western blotting, H\&E staining, and immunohistochemistry.

Conclusions: Taken together, this bionic composite scaffold with osteochondrogenic factors offers a promising option for the repair of full-thickness osteochondral defects.

\section{Background}

Because of its complex structure, the articular cartilage is difficult to completely self-repair after injury. According to statistics, 250000 people in the United States receive cartilage repair operations every year [1]. Therefore, the means by which defects of the articular cartilage could be repaired have important clinical value and social significance. At present, the main clinical treatment methods include microfracture technology, chondrocyte transplantation, and osteochondral transplantation, each of which has its own limitations [2]. Furthermore, defects of the cartilage could cause pain and dysfunction of joints, and often involve subchondral bone, as the cartilage and bone tissues have different physiological structures and biological characteristics [3]. Hence, the repair of defects of osteochondral nature still faces great challenges [4].

As one of the three major elements employed for tissue engineering, scaffolds provide the favorable microenvironment of spatial structure and mechanical strength, facilitate seed cells adhesion, proliferation, and differentiation, carry bioactive substances, and assist the sustained release, thus giving new hope for the repair of cartilage defects [5]. Among several biomaterials, the injectable hydrogel has been shown to be more suitable for cartilage biomimetic scaffolds, contributing to this area [6, 7]. Up to 
now, some research has been focused on the partial cartilage defect repair by the homogeneous scaffolds like hydrogels, ignoring the effects on the subchondral bone. However, it could be hard to satisfy the requirement for simultaneous repair of cartilage and subchondral bone using the monolayer scaffolds alone.

With the continuous development of tissue engineering technologies, new ideas have emerged in the repair of osteochondral defects [8]. Currently, the development of scaffolds (often biphasic) mainly focuses on the simulation of the natural articular cartilage structure. The biphasic scaffold's upper layer mostly consists of low-strength hydrogel for cartilage regeneration, whereas their lower layer is often composed of high-strength porous scaffolds for subchondral bone repair. Several biphasic scaffolds, including natural and/or synthetic materials with or without cells and/or growth factors, have been studied and achieved great progress on the repair of osteochondral defects. However, the following challenges limit their clinical applications: 1) the ability of seed cells to proliferate and differentiate on osteochondral composite scaffolds is still not optimal, leading to low tissue quality; 2 ) the between mechanical strength and the degradation rate of scaffolds results in unsatisfactory mechanical properties and varying cartilage formation rate; and 3) the insufficient binding force between these two layers and their poor integration within the host interface leads to stratification.

Biphasic scaffolds with different mechanical strengths, degradation rates, and osteochondral factors and endogenous and exogenous cells show promising potential for the simultaneous cartilage and bone repair. For instance, polypeptide thermogel with versatile biofunction not only has the advantage of not generating acid products during degradation and good biocompatibility, but also carries adjustable mechanical properties, not available in natural gel [9]. Particularly, the MPEG-block-poly(L-valine) (mPEG$b$-PLV) thermogel with excellent cytocompatibility could provide a 3D meshwork for cell adhesion and proliferation [10], which can be used as the optimal material in the upper layer. Meanwhile, hydroxyapatite (HA) has been one of the most widely used bone repair materials because of its bone conduction and osteogenesis. However, pure HA is known to be fragile, exhibiting low fatigue resistance and poor degradation, seriously limiting its clinical application. In order to make up for the defects of HA, researchers proposed to combine HA with poly(lactide-co-glycolide) (PLGA) degradable materials to generate bone repair materials with good mechanical properties and appropriate degradation [11].

In addition to the scaffold, biological factors such as cells and growth factors also play a significant role in the repair and reconstruction of osteochondral injury. Endogenous mesenchymal stem cells (MSCs) have been shown to serve as a reservoir for the maintenance of the normal joint. However, once the cartilage is damaged, the endogenous MSCs are out of balance and insufficient to complete the required repair, thus there is a need to be supplemented by exogenous MSCs [12]. Therefore, we proposed repair of cartilage with exogenous extractive MSCs and subchondral bone with endogenous bone marrow MSCs (BMMSCs). Additionally, as a small nonprotein chondrogenic inducer molecule, kartogenin (KGN) has been suggested as a potential chondrogenic promoter of cartilage tissue engineering, functioning in cartilage protection and inhibition of osteoarthritis [13], and effectively promote the differentiation of MSCs into chondrocytes [14]. Furthermore, the rapid repair of subchondral bone is of great significance 
for the repair of cartilage. As an excellent osteogenic inducer, morphogenetic protein-2 (BMP-2) can promote the differentiation of MSCs into osteoblasts, induce bone collagen formation, and improve the quality of bone regeneration [15].

Besides, as a complex of subchondral bone and cartilage, the calcification zone should be paid more attention clinically, so as to achieve the goal of osteochondral integration. To this end, we fabricated a bionic biphasic composite scaffold with on-demand osteochondrogenic factors (Fig. 1). To achieve optimal repair of the articular surface cartilage, KGN and extractive BMMSCs were introduced into the $\mathrm{mPEG}-b$-PLV thermogel, as the upper layer. A PLGA/HA porous scaffold was immobilized with BMP-2 as the bottom layer to facilitate the therapeutic effect on the defects of the subchondral bone. Moreover, the intermediate interface was the firm combination of these 2 layers to facilitate the reconstruction of the calcification zone. The microstructure of the composite biphasic scaffold was confirmed by microcomputed tomography (micro-CT) and confocal laser scanning microscopy (CLSM). The mPEG- $b$-PLV thermogel and PLGA/HA scaffold exhibited appropriate degradation rate and sustained release of KGN and BMP-2, and provided special microenvironment for the regeneration of the cartilage and subchondral bone, respectively. Finally, composite scaffolds embedded with BMMSCs and osteochondrogenic factors were transplanted into full-thickness osteochondral defects of rabbits for 3 and 6 months. Gross evaluation, micro-CT, and magnetic resonance imaging (MRI) exhibited excellent osteochondral repair in the treatment group at 6 months post operation. Additionally, HE staining, immunohistochemistry, and western blot analysis further demonstrated that the composition of the regenerated osteochondral tissue was highly resembling that of the adjacent normal cartilage and bone area. On the whole, the bionic biphasic composite scaffold might provide the basis for promising projects and advancements in the engineering of osteochondral tissues.

\section{Materials And Methods}

Materials

mPEG-NH ${ }_{2}$, L-valine (L-Val), triphosgene (TPG), and tetrahydrofuran (THF) were purchased from Shanghai Yeexin Biochem \& Tech Co., Ltd. (Shanghai, PR China). PLGA and HA were obtained from Sinobiomaterials (Changchun, China). KGN was synthesized by 0-phthalic anhydride and 4aminobiphenyl according to the previous experiment. BMP-2 was obtained from Sigma-Aldrich (Steinheim, Germany). H\&E, Masson and Safranin-O/fast green staining solutions, as well as elastase, were all purchased from Sigma-Aldrich (St. Louis, MO, USA). Penicillin-streptomycin and trypsin-EDTA (0.05\% trypsin and 0.02\% EDTA) solutions, low-glucose Dulbecco's Modified Eagle's medium (LG-DMEM), and fetal bovine serum (FBS) were from Gibco (Grand Island, NY, USA). Materials used in sodium dodecyl sulfate-polyacrylamide gel electrophoresis (SDS-PAGE) were bought from Bio-Rad (Hercules, CA, USA). Total protein extraction buffer was obtained from Transgen Biotech (Beijing, China). The chemiluminescence (ECL) kit and polyvinylidene difluoride (PVDF) membranes were bought from Millipore (Burlington, MA, USA). The RNA reverse transcription kit and the real-time reverse transcriptionpolymerase chain reaction (RT-PCR) kit were purchased from Takara (Shiga, Japan). Primary antibodies 
were purchased from Bioss (Beijing, China). Tissue staining kits containing horseradish peroxidase (HRP)-conjugated anti-rabbit secondary antibodies and 3,3'-diaminobenzidine (DAB) as a chromogen were obtained from Lab Vision (Shanghai, PR China).

\section{Preparation of mPEG-b-PLV Copolymer}

L-valine-N-carboxyanhydride (L-Val NCA) was prepared by mixing and stirring TPG and L-Val in THF solution at $55^{\circ} \mathrm{C}$. After that, mPEG- $b$-PLV copolymer was obtained by ring-opening polymerization of recrystallized L-Val NCA initiated with $\mathrm{mPEG}-\mathrm{NH}_{2}$ at room temperature (RT) for 3 days.

\section{Phase Diagram of mPEG-b-PLV Thermogel}

The sol-gel transition behavior of mPEG- $b$-PLV in phosphate-buffered saline (PBS, pH 7.4) was tested by tube inversion method with a rising temperature of $2^{\circ} \mathrm{C}$ per step. First, samples with concentrations ranging from 5.0 to $9.0 \mathrm{wt} \%$ were dissolved in PBS. After stirring at $0^{\circ} \mathrm{C}$ for $12 \mathrm{~h}$, the copolymer solution $(0.2 \mathrm{~mL})$ was transferred into the tube with an inner diameter of $10.0 \mathrm{~mm}$. The sol-gel transition temperature was recorded if the flow was not observed within $30 \mathrm{~s}$ after the inverted test tube.

\section{Preparation of PLGA/HA Scaffold}

The PLGA was dissolved in chloroform, and then HA powder was added in a ratio of $10 \%$ by weight of the composite material (HA/PLGA), and the mixture was thoroughly mixed; the above material was sedimented with a solution of absolute ethanol to obtain a solid mixture material. Thereafter, a salt (having a diameter of about $200 \mu \mathrm{m}$ ) used as a porogen was added to the above-mixed material, and the mixture was stirred and mixed in an internal mixer to prepare a composite of the material and the salt particles. Then, it was placed in a hydraulic press, heated, and pressed to form a thin plate. After cooling, the obtained material was taken out and immersed in deionized water for 1 day to completely remove the porogen, and finally dried in a clean bench to obtain the PLGA/HA scaffold.

\section{Preparation and Microstructure of Biphasic Scaffolds}

The biphasic scaffolds consisted of the mPEG- $b$-PLV thermogel (7.0 wt\%), porous PLGA/HA scaffold, and their permeable binding zone. Scaffolds were frozen with liquid nitrogen and quickly brittle broken to obtain the cross-section. Accordingly, the microstructure of these scaffolds was observed by micro-CT (Bruker, Belgium), scanning electron microscopy (SEM, Hitachi, Japan), and CLSM (Zeiss, Jena, Germany).

Preparation of Inducible Factor Loaded Hydrogel and Coated Scaffolds

The KGN-entrapped mPEG- $b$-PLV hydrogel was prepared by dissolving mPEG- $b$-PLV copolymer and KGN $(1 \mu \mathrm{M})$ in PBS at $4^{\circ} \mathrm{C}$ under gentle stirring. BMP-2 was thoroughly dissolved in PBS $(0.01 \mathrm{M} ; \mathrm{pH} 7.4)$ at a concentration of $10.0 \mathrm{mg} / \mathrm{mL}$. The PLGA/HA scaffold was fabricated into a circular scaffold with a diameter of $5 \mathrm{~mm}$ and a thickness of $2 \mathrm{~mm}$, and placed in a safety bottle, which was evacuated, thereby 
generating negative pressure inside the scaffold. The BMP-2 solution was fully immersed the scaffold under negative pressure after injection. The scaffold was shaken on a shaking bed 50 times per min for 10 min, thereby allowing the BMP-2 to be uniformly coated on the pore surface. After freeze-drying, the scaffold was stored for further use.

In Vitro Degradation of the mPEG-b-PLV Thermogel and PLGA/HA Scaffold

The copolymer solution $(7.0 \mathrm{wt} \%, 0.5 \mathrm{~mL})$ was transferred to a small bottle with an inner diameter of 16 $\mathrm{mm}$, and divided into a PBS and elastase (PBS containing $0.2 \mathrm{mg} / \mathrm{mL}$ elastase) group after gelation. Subsequently, $1 \mathrm{~mL}$ of degradation solution was added on top of the gel and changed at a predetermined time point, and following that the residual mass was weighted.

After the PLGA/HA scaffold was trimmed to a cylinder $(5 \times 5 \times 2 \mathrm{~mm})$, it was divided into 3 groups: Scaffold, Scaffold + elastase, and Scaffold ${ }^{\mathrm{BMP}-2}$. Respectively, $2 \mathrm{~mL}$ degradation medium was added to the vial of each group, and replaced at a predetermined time point. Finally, the degradation rate was calculated. The cross section of the scaffold was evenly sprayed with gold, and the pore size and pore distribution of the scaffolds were observed under SEM.

In Vivo Degradation and Biocompatibility of the mPEG-b-PLV Thermogel and PLGA/HA Scaffold

The thermogel $(0.5 \mathrm{~mL})$ was injected into the subcutaneous tissue of Sprague-Dawley (SD) rats. Rats were sacrificed at $30 \mathrm{~min}, 1$ week, 3 weeks, and 5 weeks after gelation. The external phase was taken to observe the degradation behavior of the gel. Then, the skin tissue around the gel was fixed and subjected to pathological sectioning to observe local inflammatory conditions.

The PLGA/HA scaffold was implanted into the muscular space of the hind limb of the rat. Rats were sacrificed in the predetermined time after operation. The residual scaffold was weighed and the degradation rate of the scaffold was calculated by comparing with the preoperational (original) weight. The muscle tissue at the contact site of the scaffold was fixed and stained with H\&E staining.

KGN Release from mPEG-b-PLV Thermogel and BMP-2 Release from PLGA/HA Scaffold in Vitro

In order to verify the ability of the MPEG- $b$-PLV thermogel for controlled release, we tested the in vitro release of KGN. The hydrogel solution containing $0.5 \mathrm{~mL} \mathrm{KGN}(200 \mu \mathrm{g} / \mathrm{mL})$ was transferred to a vial, and $1 \mathrm{~mL}$ PBS was added to the MPEG- $b$-PLV thermogel. At the predetermined time point, the released liquid was transferred to a centrifuge tube for cryopreservation and fresh PBS was added to the vial. Finally, the concentration of KGN in the released medium was determined using the UV analyzer (Hitachi, Japan).

The controlled release ability of the PLGA/HA porous scaffolds was demonstrated by in vitro release experiments of BMP-2. First, BMP-2 was labeled with FITC, and then its release was detected. The specifiC steps were as follows: (1) $5 \mathrm{mg} \mathrm{BMP-2}$ and $0.5 \mathrm{mg}$ FITC were mixed and dissolved, and then centrifuged in an ultrafiltration tube $\left(2800 \mathrm{~g}, 4^{\circ} \mathrm{C}, 5 \mathrm{~min}\right)$ to obtain a FITC-labelled BMP-2 solution $(0.5 \mathrm{mg} / \mathrm{mL})$; (2) The PLGA/HA scaffold was transferred to a vial, add $2 \mathrm{~mL}$ PBS was added on top. Then, the released 
medium was put into a centrifuge tube at a predetermined time point for freezing and storage, and fresh PBS was added to the vial. Finally, the concentration of BMP-2 in the released liquid was determined using the full wavelength multifunctional enzyme labeling instrument (Tecan, Switzerland).

Isolation and Culture of BMMSCs

BMMSCs were isolated from the femur and tibia of a 1-month-old male New Zealand rabbit. Briefly, after anesthetization with pentobarbital $(50 \mathrm{mg} / \mathrm{kg})$ intravenous injection, bone marrow $(10 \mathrm{ml})$ was extracted using a 23-gauge needle attached to a $10-\mathrm{ml}$ syringe containing heparin $(0.2 \mathrm{ml} ; 1 \mathrm{wt} \%)$. Cells were homogenized, centrifuged, repeatedly washed, and finally incubated with complete LG-DMEM medium $(10 \mathrm{ml})$. After $24 \mathrm{~h}$, the medium was replaced with fresh LG-DMEM medium, and non-adherent cells were removed, digested with trypsin-EDTA at $37^{\circ} \mathrm{C}$ for $3 \mathrm{~min}$, and passaged. Third-generation BMMSCs were collected and implanted into in vivo cartilage defects.

Animal Procedure

First, 3-month-old male New Zealand white rabbits (about $2.5 \mathrm{~kg}$ ) were anesthetized, and after the knee joint was cut and the femoral condyle was exposed, an osteochondral defect with a diameter of $5 \mathrm{~mm}$ and a depth up to the medullary cavity was made by drilling in the femoral condyle. Then, the rabbits were implanted with different scaffolds and divided stochastically into 4 groups of 8 animals each: control group (Control); group receiving composite scaffolds: thermogel and PLGA/HA (Gel/Scaffold); group receiving composite scaffolds encapsulated with MSCs in the gel (Gel-MSCs/Scaffold), and group

receiving the Gel-MSCs/Scaffold co-loaded with KGN and BMP-2 (Gel ${ }^{\mathrm{KGN}}-\mathrm{MSC}$ /Scaffold ${ }^{\mathrm{BMP}-2}$ ). Relevant tests were carried out at 3 and 6 months post operation.

\section{Gross Observation of Cartilage Repair}

At the third and sixth month, some rabbits were euthanized, and their femoral condyles were collected. The repair of the cartilage was evaluated according to the ICRS standard score (Table S1).

\section{Micro-computed Tomography}

The obtained femoral condyle samples were photographed and analyzed with micro-CT. Parameters were set to $1000 \mathrm{~K}$ resolution for X-ray scanning. The thickness of each layer was $0.1 \mu \mathrm{m}$. After that, the CT-An analysis software (Bruker, Belgium) was used for correction of parameters and reconstruction, followed by analysis of the bone mass.

Magnetic Resonance Imaging

To evaluate the morphology of the generated neo-cartilage, rabbits underwent MRI scanning at 6 months post operation on a TIM Trio 3T (T) MRI scanner (Siemens, Erlangen, Germany) using a small animalspecific knee coil. Accordingly, the T2 and T2* values were analyzed. 
Part of the femoral condyle was fixed with $4 \%$ paraformaldehyde for $48 \mathrm{~h}$, then decalcified with $10 \%$ EDTA solution, dehydrated, paraffin embedded, and histologically sectioned. The thickness of the sections was $5 \mu \mathrm{m}$. The pathology of H\&E, Masson's trichrome, and Safranin-0/fast green stained samples was evaluated by observation under a light microscope, and quantified using the ICRS histological score (Table S2).

Immunohistochemistry

In order to further validate the effect of osteochondral repair, we performed immunohistochemistry tests. Briefly, paraffinized tissue sections $(5 \mu \mathrm{m})$ were dewaxed in xylene, rehydrated in ethanol, and treated with $3 \% \mathrm{H}_{2} \mathrm{O}_{2}$ in absolute methanol for $30 \mathrm{~min}$. Next, the sections were immersed in citrate buffer $(\mathrm{pH} 6.0)$, boiled for $10 \mathrm{~min}$, and cooled to room temperature. The slides were blocked with normal goat serum for 30 min and incubated with rabbit polyclonal anti-DGAT2 antibodies (1:200 dilution; Santa Cruz Biotechnology, Santa Cruz, CA, USA) at $4^{\circ} \mathrm{C}$ overnight. The next day, the samples were submitted to $A B C$ (kit from Zhongshan Golden Bridge Biotechnology, Beijing, China) (biotin: 1 h, streptavidin: 30 min; $37^{\circ} \mathrm{C}$ ), followed by incubation with DAB for 1 min. Counterstaining was performed with Mayer's hematoxylin. Finally, immunohistochemistry signals were detected via CLSM.

\section{Western Blotting and RT-PCR Analysis}

The expression of cartilage and subchondral bone proteins was examined by western blotting as previously described [16]. In detail, the cartilage and subchondral bone of each group were lysed with total protein extraction buffer, and lysates were centrifuged at $12000 \mathrm{~g}$ for $10 \mathrm{~min}$ at $4^{\circ} \mathrm{C}$. Then they were fractionated by SDS-PAGE and transferred onto PVDF membranes. After being blocked with $5 \% \mathrm{skim}$ milk for $1 \mathrm{~h}$, membranes were incubated overnight with primary antibodies. After that, blots were incubated with secondary antibodies (1:5000; Lab Vision, Shanghai, China) for 40 min and then activated by an enhanced ECL kit and evaluated using the Image $J$ software (National Institutes of Health, Bethesda, MD, USA).

The levels of the mRNA expression of chondrogenic and osteogenic specific genes were evaluated using RT-PCR. Briefly, $1 \mathrm{~mL}$ of Trizol was added to the bone and cartilage tissues to extract total RNA, which was then reverse-transcribed; CDNAs were obtained according to the instructions of the RT-PCR kit. Then, the amplification and fusion curves of RT-PCR were confirmed, and the standard curve was generated during PCR quantification. Primer sequences are shown on Table S3.

Statistical Analysis

The values of all counting results were given in the form of mean \pm standard deviation (SD), and all experimental results were repeated 3 times. The statistical differences of the study results were analyzed in accordance with the $t$-test utilizing the SPSS 24.0 software (Chicago, Illinois, USA). ${ }^{*}<0.05$ was 
considered statistically significant, whereas ${ }^{\star \star} P<0.01$, and ${ }^{* \star *} P<0.001$ were considered highly statistically significant.

\section{Results And Discussion}

Preparation and Characterizations of Bionic Biphasic Composite Scaffold

The MPEG- $b$-PLV polypeptide copolymer was generated via the ring-opening polymerization of $N$-carboxy anhydrides of L-valine, using mPEG- $\mathrm{NH}_{2}$ as the initiator [10] (Fig. 2A). Different mPEG- $b$-PLV copolymer concentrations experienced the transition between the sol-gel states; as the temperature rose from 4 to $70^{\circ} \mathrm{C}$, the sol-gel temperature decreased from 27.2 to $7.1^{\circ} \mathrm{C}$, while the concentration of the polymer increased from 5.0 to $9.0 \mathrm{wt} \%$ (Fig. 2B). Among them, the $7.0 \mathrm{wt} \%$ copolymer with an appropriate gelling temperature $\left(16.7^{\circ} \mathrm{C}\right)$ was considered to be the most suitable for tissue engineering and the delivery of inducible factors [17]. Thus, it was used in subsequent experiments. For assessment of their microstructure, both the Gel, Scaffold and Gel/Scaffold were subjected to analysis by CLSM and microCT (Fig. 2C). As expected, pores provide the space for the growth of cells, and the pore size of the Gel was shown to be smaller than that of the porous Scaffold, making them suitable for the cartilage and bone repair, respectively [18]. Moreover, as demonstrated by CLSM, there was a penetration noted between the Gel (green) and the Scaffold (red) forming a middle layer for the simulation of the calcification zone of the cartilage. Additionally, micro-CT scans showed the shape and spatial relationships of the Gel/Scaffold. The Gel was tightly bound to the Scaffold, mimicking the cartilage layer firmly anchored to the subchondral bone layer [19].

In vitro and in vivo degradation experiments were performed to assess the degradation properties of the mPEG- $b$-PLV thermogel and PLGA/HA scaffold. Of note, the degradation profiles of the Gel/Scaffold are important for optimal neo-tissue growth; rapid degradation impairs the formation of the extracellular matrix, whereas slow degradation restricts tissue restoration [20]. As shown in Fig. 2D, at 30 days, the in vitro residual mass rate of the Gel group $(48.4 \pm 0.1 \mathrm{wt} \%)$ was higher $(P<0.05)$ than that of the Gel + elastase group (24.7 $\pm 3.6 \mathrm{wt} \%)$ owing to the catalytic effect of the enzyme [21]. Similarly, at 60 days, compared with the Scaffold group $(86.5 \pm 1.6 \mathrm{wt} \%)$, the residual mass rate of the Scaffold + elastase $(76.1 \pm 1.4 \mathrm{wt} \%)$ and Scaffold ${ }^{\mathrm{BMP}-2}(79.8 \pm 1.0 \mathrm{wt} \%)$ groups showed faster release (Fig. 2E) [22]. During the period from 0 day until 2 months, the pore size of the Scaffold ${ }^{\mathrm{BMP}-2}$ was noted to be gradually increased in in vitro degradation tests, from $180 \pm 12$ to $240 \pm 14 \mu \mathrm{m}$, thus facilitating the growth of the bone [23] (Fig. 2F).

As depicted in Fig. S1A, at the predetermined interval, the shape of the in vivo mPEG- $b$-PLV thermogel was photographed, and shown to be almost absorbed at 5 weeks post injection. These results are consistent with our previous study in cartilage tissue engineering; the degradation rate was matched with that of new cartilage regeneration, facilitating matrix deposition [24]. Moreover, the degradation rate of the in vivo thermogel was faster than that of the in vitro counterpart due to the complex environment in the body [25]. Similarly, the in vivo PLGA/HA scaffold was shown to be respectively absorbed and degraded 
as time passed, and the residual amount of the PLGA/HA scaffold was more than $39.5 \%$ at 8 weeks (Fig. S1B); this suggests that the scaffolds allow for up to 3 months of bone repair in vivo [26]. These results confirm that the in vivo degradation rates of the Gel and Scaffold are consistent with cartilage and bone formation, providing a basis for the gradual substitution with newborn tissues [27].

To assess the biocompatibility of these constructs, we performed H\&E staining of the subcutaneous tissue of the implanted thermogel. As noted, the inflammatory reaction reached a peak with massive amounts of neutrophils at 1 week, and then gradually decreased (Fig. S1C). A similar phenomenon could also be seen in the biocompatibility test of the PLGA/HA scaffold, where a large number of granulocytes were observed to gather in the field of view at 2 weeks, with the inflammatory reaction subsiding at 4 weeks (Fig. S1D). The in vivo degradation test demonstrated the good biocompatibility of both the mPEG$b$-PLV thermogel and PLGA/HA scaffold. As shown in Fig. 1G, the in vitro release profile of KGN in the mPEG- $b$-PLV thermogel exhibited a steady and sustained way of release, which is consistent with previous findings [28]. The sustained release is crucial for KGN to promote cartilage formation and protection in vivo. On the other hand, the release profile of BMP-2 in the PLGA/HA scaffold showed an initial relatively quick release at the first 4 days, followed by a sustained release (Fig. $2 \mathrm{H}$ ). Quantitively, the cumulative amount of KGN released from the MPEG- $b$-PLV thermogel $(75.4 \%)$ was similar to the cumulative amount of BMP-2 released from the PLGA/HA scaffold (73.1 \%) at 20 days, indicating a simultaneous promotion of osteochondral repair [29].

Macroscopic Observation and Quantification of Cartilage Repair

Evaluation of gross appearance is the most direct and effective way to evaluate the effect of cartilage repair. At the predetermined interval, the femoral condyles of the 4 groups were obtained and photos were captured. The macroscopic view of cartilage repair at 3 and 6 months post operation is shown in Fig. 3A. At 3 months, the Control group was filled with soft fibrous tissue, accompanied by cartilage degeneration. In contrast, the Gel/Scaffold group was observed to be filled with a sunken repair surface, while part of neo-cartilage was formed in the Gel-MSCs/Scaffold group. Interestingly, the repair tissue of the GelKGN MSCs/Scaffold ${ }^{\mathrm{BMP}-2}$ group was noted to be forming more hyaline cartilage and result in more integration. At 6 months, compared with the Control group, improved gross results were found in all experimental groups, with the repaired effect of the experimental groups demonstrated to be significantly upregulated $(P<0.001)$. The Control group showed obvious arthritis and joint deformation, whereas the Gel ${ }^{\mathrm{KGN}}$ MSCs/Scaffold ${ }^{\mathrm{BMP}-2}$ group revealed the best regeneration of hyaline cartilage with a smooth wellintegrated surface. A previous study has reported better osteochondral repair in biphasic scaffolds with osteochondral factors, therefore supporting our findings [30].

To quantitively assess the macroscopic view of repaired tissues, the ICRS score was recorded for each experimental group. As depicted in Fig. 3B, at 3 and 6 months, the ICRS score of the Gel-MSCs/Scaffold group was significantly higher than that of the Gel/Scaffold group $(6 \pm 0.98$ and $6.67 \pm 0.49$ versus $3.67 \pm$ 0.64 and $5.33 \pm 0.53, P<0.01$ ), and the Gel ${ }^{\mathrm{KGN}}-\mathrm{MSCs} / \mathrm{Scaffold}^{\mathrm{BMP}-2}$ group exhibited a vitally better score $(10.6 \pm 0.46$ and $11.32 \pm 0.41, P<0.01)$ than that of the aforementioned 2 groups. 
We performed micro-CT scan to evaluate the regenerated subchondral bone. According to the coronal and transverse CT images, there were gradually increased degrees of bone repair in the 4 groups at 3 months. Bone repair was significantly impaired in the Control group $(P<0.001)$, whereas the Gel/Scaffold and Gel$\mathrm{MSCs} / \mathrm{Scaffold}$ groups were shown to be filled with partially newly formed bone, and the Gel ${ }^{\mathrm{KGN}}$. MSCs/Scaffold ${ }^{\text {BMP-2 }}$ group exhibited the best state of repair among all.

CT images have been previously used to observe bone regeneration [31]. At 6 months, the defect area of the Gel/Scaffold and Gel-MSCs/Scaffold groups were filled with uncontrollable high density bone tissue, exhibiting little trabecular structure. In contrast, in the Gel ${ }^{\mathrm{KGN}}-\mathrm{MSC} / \mathrm{Sc}$ affold ${ }^{\mathrm{BMP}-2}$ group we could observe an obvious effect of bone remodeling, leading to the formation of trabecular structure similar to the surrounding cancellous bone, which is of great significance for the function of bone mobility [32]. As we know, the process of bone formation includes osteogenesis and reconstruction periods; in the process of reconstruction, the redundant bone can be continuously removed, which is helpful for the formation of the skeleton and the control of bone mass density. Consequently, this interesting finding might be due to osteoinductive factors accelerating the process of bone repair [33]. Analysis of bone volume (BV) also supported this conclusion (Fig. 3D). Quantitively, the calculated BV $(26.15 \pm 1.3)$ of the Gel ${ }^{\mathrm{KGN}}$ MSCs/Scaffold ${ }^{\mathrm{BMP}-2}$ group at 3 months was significantly $(P<0.001)$ higher than that in other groups. Consecutively, at 6 months the BV value was decreased to $15.47 \pm 0.8$.

Magnetic Resonance Imaging Examination and Quantification of Repaired Cartilage

We employed MRI scan to evaluate the regenerated cartilage. As depicted in Fig. 3E, at 6 months, structures of the synovium could be observed all around the defect area of the Control group, whereas the defect area of the Gel/Scaffold group was partially filled with newly formed tissues, the cartilage signal was interrupted in the above two groups. Moreover, the defect area of the Gel-MSCs/Scaffold group was revealed to exhibit lower signal intensity than normal hyaline cartilage. However, the regenerated cartilage in the Gel ${ }^{K G N}-M S C s / S c a f f o l d{ }^{B M P-2}$ group was thicker than the Gel-MSCs/Scaffold group and exhibited signals similar to those for normal hyaline cartilage. Previous MRI scans have showed an increased signal in the experimental group, thus supporting this result [34]. Using T2 mapping assessment, the T2 signal of the Gel ${ }^{\mathrm{KGN}}-\mathrm{MSC}$ /Scaffold ${ }^{\mathrm{BMP}-2}$ group was shown to resemble that of normal cartilage, and appeared better than that of the other groups, which is consistent with previous findings [35]. Hence, our MRI imaging analysis displayed an excellent efficiency for cartilage repair in the Gel ${ }^{\mathrm{KGN}}$. MSCs/Scaffold ${ }^{\text {BMP-2 }}$ group.

Histological Evaluation and Immunohistochemistry of Repaired Tissues

Histological detection techniques, including H\&E, Masson, and Safranin O/fast green staining, as well as COL I and COL II immunohistochemistry staining, were used to evaluate the quality of the repaired tissues. As depicted in Fig. 4A, the Control group exhibited a disorganized structure, with fibrous tissues 
at 3 and 6 months. Whereas compared with other groups, the Gel ${ }^{\mathrm{KGN}}-\mathrm{MSC} / \mathrm{Scaffold}{ }^{\mathrm{BMP}-2}$ group was shown to accomplish an excellently repaired regenerated cartilage, with a reconstructed calcified layer. Interestingly, as time went on, the number of blue stained nuclei decreased, and the extracellular matrix increased in the Gel ${ }^{\mathrm{KGN}}-\mathrm{MSC}$ /Scaffold ${ }^{\mathrm{BMP}-2}$ group. Additionally, we noted a discontinued cartilage in the Gel-MSCs/Scaffold group at 3 months, whereas the defect area of the Gel-MSCs/Scaffold group was fully filled with neo-cartilage at 6 months. Consistent with previously reported findings, our histological results suggest the promising potential of bionic biphasic composite scaffold with osteochondrogenic factors on-demand [36]. Quantitively, the ICRS histological score of repaired tissues (Fig. 4B) in the Gel $^{\mathrm{KGN}}-\mathrm{MSC}$ /Scaffold ${ }^{\mathrm{BMP}-2}$ group was significantly higher than that of the Gel-MSCs/Scaffold group ( $P$ $<0.01$ ), while the score in the Gel-MSCs/Scaffold group was significantly higher than that of the Gel/Scaffold group $(P<0.01)$.

The results of $\mathrm{COL}$ I and COL II immunohistochemistry are shown in Fig. 4B and 4C, respectively. At 3 and 6 months, the Gel ${ }^{\mathrm{KGN}}-\mathrm{MSCs} / \mathrm{Scaffold}{ }^{\mathrm{BMP}-2}$ group exhibited the best repair effect, which was consistent with the histological staining results. There was no obvious COL I and COL II staining in the newly formed tissues in the cartilage layer in both the Control and the Gel/Scaffold groups. On the other hand, while there was no obvious $\mathrm{COL}$ I staining in the cartilage layer in the $\mathrm{Gel}^{\mathrm{KGN}}-\mathrm{MSC}$ /Scaffold ${ }^{\mathrm{BMP}-2}$ group at 6 months, uniform staining was observed in COL II. A previous study using KGN as a chondroblast inducer has also shown that continuous release of KGN can promote type 2 collagen deposition [37].

Masson staining was performed to identify the presence of collagen fibers in cartilage (COL II) and bone (COL I) tissues and evaluate the maturation of the bone matrix. As depicted in Fig. 5A, the percentage of green staining in the neo-cartilage and red staining in the regenerated bone gradually increased in each group, highest of which was observed in the Gel ${ }^{\mathrm{KGN}}-\mathrm{MSC} / \mathrm{Scaffold}{ }^{\mathrm{BMP}-2}$ group at 3 and 6 months, respectively. In detail, at 6 months both the Control and Gel/Scaffold group demonstrated an incomplete filling, with a big gap, whereas although collagen deposition was found in the Gel-MSCs/Scaffold group, it was still insufficient. However, there was plenty of collagen with uniform deposition observed in the $\mathrm{Gel}^{\mathrm{KGN}}$-MSCs/Scaffold ${ }^{\mathrm{BMP}-2}$ group. Moreover, the magnitude of collagen deposition in the Gel ${ }^{\mathrm{KGN}}$ MSCs/Scaffold ${ }^{\mathrm{BMP}-2}$ group at 6 months was greater than that at 3 months, indicating the formation of more mature bone tissues. Of note, a previous study has similarly reported collagen increase in the experimental group, indicating the enhanced formation of cartilage and subchondral bone and efficient osteochondral regeneration [17].

Safranin-0/fast green staining was performed to assess the regeneration of both cartilage and bone. As shown in Fig. 5B, there was sufficient hyaline cartilage (red), with an adequate deposition of GAG and repair of subchondral bone (blue) noted in the Gel ${ }^{K G N}-M S C s / S c a f f o l d{ }^{B M P-2}$ group, whereas a mixture of newly-formed fibrous tissue and cartilage was found in the Gel/Scaffold and Gel-MSCs/Scaffold groups. Additionally, there is a good integration between the cartilage and bone interfaces in the Gel ${ }^{\mathrm{KGN}}$ MSCs/Scaffold ${ }^{\mathrm{BMP}-2}$ group, and no evidence of excessive bone growth into the cartilage area was observed [38]. 
Western Blotting and RT-PCR Analysis of Repaired Tissues

The expression levels of special proteins and genes in the repaired tissues were analyzed by western blotting and RT-PCR, respectively.

The expression levels of the most representative proteins, including COL II, GAG, aggrecan (AGG), and SOX9 proteins in cartilage, as well as COL I, osteopontin (OPN), OCN, and ALP in subchondral bone at 6 months post operation are depicted in Fig. 6A. The depositions of COL II, GAG, AGG, and SOX9 in the cartilage were gradually upregulated in the Control, Gel/Scaffold, Gel-MSCs/Scaffold, and Gel ${ }^{\mathrm{KGN}}$. MSCs/Scaffold ${ }^{\text {BMP-2 }}$ groups, which is consistent with a previous study that reported the positive effects of KGN on the promotion of chondrogenic induction of BMMSCs and cartilage regeneration [39]. Similarly, the depositions of OCN and ALP in the subchondral bone demonstrated an increasing trend in these 4 groups. However, the depositions of OPN and COL I in the Gel ${ }^{K G N}-M S C s / S c a f f o l d{ }^{B M P-2}$ group were less than those of the Gel/Scaffold and Gel-MSCs/Scaffold groups, and the contents of the Gel ${ }^{K G N}$. MSCs/Scaffold ${ }^{\text {BMP-2 }}$ group were close to those in normal subjects. Noted, OPN is the first matrix protein, representing the formation and maturation of the matrix. With the differentiation and maturation of osteoblasts, osteoclasts begin to play a role, and the introduction of inducers might accelerate this process, followed by the decreased deposition of OPN [40]. Bone remodeling has begun at this stage and osteoclasts appear to play a role in the Gel ${ }^{\mathrm{KGN}}-\mathrm{MSCs} / \mathrm{Scaffold}{ }^{\mathrm{BMP}-2}$ group, with any extra bone formed in the healing process being decomposed, whereas the Gel/Scaffold and Gel-MSCs/Scaffold groups lacking inducible factors would still be in the process of bone repair. This might explain the decrease in the deposition of COL I in the Gel ${ }^{\mathrm{KGN}}-\mathrm{MSC} /$ Scaffold $^{\mathrm{BMP}-2}$ group [41].

Quantitively, the expression levels of COL II, GAG, AGG, and SOX9 $(0.91 \pm 0.04,0.93 \pm 0.03,1.01 \pm 0.02$, and $0.65 \pm 0.05$, respectively) and the expression levels of OCN, and ALP $(1.03 \pm 0.03$ and $1.08 \pm 0.07)$ in the Gel ${ }^{\mathrm{KGN}}-\mathrm{MSCS} /$ Scaffold ${ }^{\mathrm{BMP}-2}$ group were significantly $(P<0.05)$ higher than those of other groups (Fig. 6B). Whereas, the expression levels of COL I and OPN in the Gel ${ }^{\mathrm{KGN}}{ }_{-M S C s} / S c a f f o l d{ }^{\mathrm{BMP}-2}$ group were lower than those of the Gel/Scaffold and Gel-MSCs/Scaffold groups. These results demonstrated the high expression levels of specific osteochondral proteins in the Gel $^{\mathrm{KGN}}$-MSCs/Scaffold ${ }^{\mathrm{BMP}-2}$ group exhibiting a satisfactory repair.

The expression levels of the corresponding chondrogenic (COL2A1, FAM, ACAN, and SOX9) and osteoblastic (COL 1A1, SPP1, SPARC, ALPL) marker genes at 6 months post operation were measured by RT-PCR (Fig. 6C). The expression levels of COL2A1, GAG, ACAN, and SOX9 genes of the repaired cartilage in the Gel ${ }^{\mathrm{KGN}}-\mathrm{MSC} /$ Scaffold ${ }^{\mathrm{BMP}-2}$ group were significantly upregulated $(P<0.05)$ compared with those of the other groups. These results demonstrated that the Gel ${ }^{\mathrm{KGN}}-\mathrm{MSC} / \mathrm{Scaffold}^{\mathrm{BMP}-2}$ group had the better capacity for chondrogenic differentiation and cartilage formation among all experimental groups, which is consistent with our previous findings [39]. Meanwhile, the expression levels of the SPARC and $A L P L$ genes of the engineered subchondral bone in the Gel ${ }^{K G N}-M S C s / S c a f f o l d{ }^{B M P-2}$ group were higher than those in other groups, representing the osteoblastic ability of the newly formed bone. Additionally, the 
expression levels of the COL1A1 and SPP1 genes in the Gel ${ }^{\mathrm{KGN}}-\mathrm{MSC}$ /Scaffold ${ }^{\mathrm{BMP}-2}$ group were lower than those of the no added-drug groups, consistent with the above protein expression levels.

\section{Conclusion}

To achieve optimal repair of osteochondral defects, we created a composite thermogel-scaffold in this study, by combining an mPEG- $b$-PLV thermogel with KGN/BMMSCs and a PLGA/HA porous scaffold with BMP-2. Interestingly, the synthesized composite scaffold exhibited an appropriate degradation rate and sustained release of KGN and BMP-2, thus providing special microenvironment for the reconstruction of the cartilage and the subchondral bone, respectively. More importantly, the introduction of BMMSCs and osteochondral inducible factors to these composite scaffolds resulted in them exhibiting fascinating therapeutic effects on cartilage and bone repair as demonstrated by gross evaluation and imaging examination at 6 months post operation. Additionally, the histology, immunohistochemistry, and biochemistry tests further demonstrated that the composition of this osteochondral regeneration was infinitely resembling the adjacent normal cartilage and subchondral bone. In summary, the generated bionic biphasic scaffold could provide a promising prospect for the integrated reconstruction of the cartilage and subchondral bone, which is worthy of further preclinical evaluation.

\section{Declarations}

\section{Supplementary Information}

The online version contains supplementary material available at

Additional fle 1: Figure S1. In vivo degradation of mPEG- $b$-PLV thermogel (A) and PLGA/HA scaffold (B). In vivo biocompatibility of mPEG- $b$-PLV thermogel (C) and PLGA/HA scaffold (D). E ICRS histological score. Table S1. ICRS Macroscopic Evaluation of Neocartilage. Table S2. ICRS Histological Grading Scale for Cartilage Regeneration. Table S3. Sequences of Primers Used for Real-time PCR.

\section{Acknowledgements}

Not applicable.

\section{Authors' contributions}

YBZ: Conceptualization, Methodology, Formal analysis, Investigation, Writing - Original Draft, Writing Review \& Editing.YH: Conceptualization, Methodology, Formal analysis, Investigation, Writing - Original Draft.YCP: Investigation,Writing - Review \& Editing. JL: Formal analysis.FC:Conceptualization, Writing Review \& Editing, Supervision, Project administration, Funding acquisition. All authors read and approved the fnal manuscript.

\section{Funding}


Funding for this study was supported by National Natural Science Foundation of China grants (81671804, 81701811) and Natural Science Foundation of Jilin Province (20200201322JC).

\section{Availability of data and materials}

The data used to support the fndings of this study are available from the corresponding author upon request.

\section{Ethics approval and consent to participate}

Not applicable.

\section{Consent for publication}

Not applicable.

\section{Competing interests}

The authors declare that they have no known competing financial interests or personal relationships that could have appeared to influence the work reported in this paper.

\section{Author details}

1Department of Orthopedics, The Second Hospital of Jilin University, 218 Ziqiang Street, Changchun 130041, P. R. China

2Department of Orthopedics, China-Japan Union Hospital of Jilin University, 126 Xiantai Street, Changchun 130033, P. R. China

3Department of MR, Changchun FAW General Hospital, Dongfeng Street 2643, Changchun 130011, P. R. China

\section{References}

1. F. McCormick, J.D. Harris, G.D. Abrams, R. Frank, A. Gupta, K. Hussey, H. Wilson, B. Bach, B. Cole, Trends in the surgical treatment of articular cartilage lesions in the united states: an analysis of a large private-payer database over a period of 8 years, Arthroscopy 30(2) (2014) 222-226.

2. B.J. Huang, J.C. Hu, K.A. Athanasiou, Cell-based tissue engineering strategies used in the clinical repair of articular cartilage, Biomaterials 98 (2016) 1-22.

3. T.M. Simon, D.W. Jackson, Articular cartilage: injury pathways and treatment options, Sports Med. Arthrosc. Rev. 14(3) (2006) 146-154.

4. Y.B. Zhang, X.C. Liu, L.D. Zeng, J. Zhang, J.L. Zuo, J. Zou, J.X. Ding, X.S. Chen, Polymer fiber scaffolds for bone and cartilage tissue engineering, Adv. Funct. Mater. 29(36) (2019) 1903279. 
5. C.Y. Wang, N.B. Feng, F. Chang, J.C. Wang, B.M. Yuan, Y.L. Cheng, H. Liu, J.K. Yu, J. Zou, J.X. Ding, X.S. Chen, Injectable cholesterol-enhanced stereocomplex polylactide thermogel loading chondrocytes for optimized cartilage regeneration, Adv. Healthcare Mater. 8(14) (2019) 1900312.

6. Y.B. Zhang, J.K. Yu, K.X. Ren, J.L. Zuo, J.X. Ding, X.S. Chen, Thermosensitive hydrogels as scaffolds for cartilage tissue engineering, Biomacromolecules 20(4) (2019) 1478-1492.

7. S.Q. Li, S.J. Dong, W.G. Xu, S.C. Tu, L.S. Yan, C.W. Zhao, J.X. Ding, X.S. Chen, Antibacterial hydrogels, Adv. Sci. 5(5) (2018) 1700527.

8. X. Li, J. Ding, J. Wang, X. Zhuang, X. Chen, Biomimetic biphasic scaffolds for osteochondral defect repair, Regener. Biomater. 2(3) (2015) 221-228.

9. H.J. Moon, D.Y. Ko, M.H. Park, M.K. Joo, B. Jeong, Temperature-responsive compounds as in situ gelling biomedical materials, Chem. Soc. Rev. 41(14) (2012) 4860-4883.

10. H.J. Song, G. Yang, P.S. Huang, D.L. Kong, W.W. Wang, Self-assembled PEG-poly(L-valine) hydrogels as promising 3D cell culture scaffolds, J. Mater. Chem. B 5(9) (2017) 1724-1733.

11. X.Y. Zhao, Y. Han, J.W. Li, B. Cai, H. Gao, W. Feng, S.Q. Li, J.G. Liu, D.S. Li, BMP-2 immobilized PLGA/hydroxyapatite fibrous scaffold via polydopamine stimulates osteoblast growth, Mat. Sci. Eng. C 78 (2017) 658-666.

12. F. Barry, M. Murphy, Mesenchymal stem cells in joint disease and repair, Nat. Rev. Rheumatol. 9(10) (2013) 584-594.

13. X.Z. Li, J.X. Ding, Z.Z. Zhang, M.D. Yang, J.K. Yu, J.C. Wang, F. Chang, X.S. Chen, Kartogeninincorporated thermogel supports stem cells for significant cartilage regeneration, ACS Appl. Mater. Interfaces 8(8) (2016) 5148-5159.

14. K. Johnson, S.T. Zhu, M.S. Tremblay, J.N. Payette, J.N. Wang, L.C. Bouchez, S. Meeusen, A. Althage, C.Y. Cho, X. Wu, P.G. Schultz, A stem cell-based approach to cartilage repair, Science 336(6082) (2012) 717-721.

15. S. Gromolak, A. Krawczenko, A. Antonczyk, J. Biezynski, B. Liszka, Z. Kielbowicz, A. Klimczak, Bone morphogenetic protein-2 promotes the osteogenic differentiation of sheep bone marrow-derived mesenchymal stem cells in vitro, Transplantation 103(9) (2019) S2.

16. J. Li, W. Xu, D. Li, T. Liu, Y.S. Zhang, J. Ding, X. Chen, Locally deployable nanofiber patch for sequential drug delivery in treatment of primary and advanced orthotopic hepatomas, ACS Nano $12(7)$ (2018) 6685-6699.

17. L. Li, J. Li, J. Guo, H. Zhang, X. Zhang, C. Yin, L. Wang, Y. Zhu, Q. Yao, 3D molecularly functionalized cell-free biomimetic scaffolds for osteochondral regeneration, Adv. Funct. Mater. 29(6) (2019) 1807356.

18. X. He, H. Fang, D.J. Gosztola, Z. Jiang, P. Jena, W.N. Wang, Mechanistic insight into photocatalytic pathways of MIL-100(Fe)/TiO2 composites, ACS Appl. Mater. Interfaces 11(13) (2019) 12516-12524.

19. M. Sartori, S. Pagani, A. Ferrari, V. Costa, V. Carina, E. Figallo, M.C. Maltarello, L. Martini, M. Fini, G. Giavaresi, A new bi-layered scaffold for osteochondral tissue regeneration: In vitro and in vivo preclinical investigations, Mat. Sci. Eng. C 70 (2017) 101-111. 
20. P. Duan, Z. Pan, L. Cao, Y. He, H. Wang, Z. Qu, J. Dong, J. Ding, The effects of pore size in bilayered poly(lactide-co-glycolide) scaffolds on restoring osteochondral defects in rabbits, J. Biomed. Mater. Res. Part A 102(1) (2014) 180-92.

21. H. Renata, Z.J. Wang, F.H. Arnold, Expanding the enzyme universe: accessing non-natural reactions by mechanism-guided directed evolution, Angew. Chem. Int. Edit. 54(11) (2015) 3351-3367.

22. B. Li, J.M. Davidson, S.A. Guelcher, The effect of the local delivery of platelet-derived growth factor from reactive two-component polyurethane scaffolds on the healing in rat skin excisional wounds, Biomaterials 30(20) (2009) 3486-3494.

23. C. Matta, R. Zakany, Challenges in engineering osteochondral tissue grafts with hierarchical structures, Expert. Opin. Biol. Th. 15(11) (2015) 1599-1599.

24. A.J. Neumann, T. Quinn, S.J. Bryant, Nondestructive evaluation of a new hydrolytically degradable and photo-clickable PEG hydrogel for cartilage tissue engineering, Acta Biomater. 39 (2016) 1-11.

25. Z. Li, J. Cao, B. Hu, H. Li, H. Liu, F. Han, Z. Liu, C. Tong, S. Li, Studies on the in vitro and in vivo degradation behavior of amino acid derivative-based organogels, Drug. Dev. Ind. Pharm. 42(11) (2016) 1732-1741.

26. J.H. Huang, X. Xia, Q. Zou, J.Q. Ma, S.E. Jin, J.D. Li, Y. Zuo, Y.B. Li, The long-term behaviors and differences in bone reconstruction of three polymer-based scaffolds with different degradability, J. Mater. Chem. B 7(48) (2019) 7690-7703.

27. A. Naik, D.V. Shepherd, J.H. Shepherd, S.M. Best, R.E. Cameron, The effect of the type of HA on the degradation of PLGA/HA composites, Mat. Sci. Eng. C 70 (2017) 824-831.

28. H. Song, P. Yang, P. Huang, C. Zhang, D. Kong, W. Wang, Injectable polypeptide hydrogel-based codelivery of vaccine and immune checkpoint inhibitors improves tumor immunotherapy, Theranostics 9(8) (2019) 2299-2314.

29. S. Lu, J. Lam, J.E. Trachtenberg, E.J. Lee, H. Seyednejad, J.J.J.P. van den Beucken, Y. Tabata, M.E. Wong, J.A. Jansen, A.G. Mikos, F.K. Kasper, Dual growth factor delivery from bilayered, biodegradable hydrogel composites for spatially-guided osteochondral tissue repair, Biomaterials 35(31) (2014) 8829-8839.

30. X. Liu, Y. Chen, A.S. Mao, C. Xuan, Z. Wang, H. Gao, G. An, Y. Zhu, X. Shi, C. Mao, Molecular recognition-directed site-specific release of stem cell differentiation inducers for enhanced joint repair, Biomaterials 232 (2020) 119644.

31. J. Radhakrishnan, A. Manigandan, P. Chinnaswamy, A. Subramanian, S. Sethuraman, Gradient nanoengineered in situ forming composite hydrogel for osteochondral regeneration, Biomaterials 162 (2018) 82-98.

32. R. Huiskes, R. Ruimerman, G.H. van Lenthe, J.D. Janssen, Effects of mechanical forces on maintenance and adaptation of form in trabecular bone, Nature 405(6787) (2000) 704-706.

33. P. Papageorgiou, Q. Vallmajo-Martin, M. Kisielow, A. Sancho-Puchades, E. Kleiner, M. Ehrbar, Expanded skeletal stem and progenitor cells promote and participate in induced bone regeneration at subcritical BMP-2 dose, Biomaterials 217 (2019) 119278. 
34. Z. Man, X. Hu, Z. Liu, H. Huang, Q. Meng, X. Zhang, L. Dai, J. Zhang, X. Fu, X. Duan, C. Zhou, Y. Ao, Transplantation of allogenic chondrocytes with chitosan hydrogel-demineralized bone matrix hybrid scaffold to repair rabbit cartilage injury, Biomaterials 108 (2016) 157-167.

35. H. Huang, X. Zhang, X. Hu, Z. Shao, J. Zhu, L. Dai, Z. Man, L. Yuan, H. Chen, C. Zhou, Y. Ao, A functional biphasic biomaterial homing mesenchymal stem cells for in vivo cartilage regeneration, Biomaterials 35(36) (2014) 9608-9619.

36. J. Chen, H. Chen, P. Li, H. Diao, S. Zhu, L. Dong, R. Wang, T. Guo, J. Zhao, J. Zhang, Simultaneous regeneration of articular cartilage and subchondral bone in vivo using MSCs induced by a spatially controlled gene delivery system in bilayered integrated scaffolds, Biomaterials 32(21) (2011) 47934805.

37. H.C. Wu, L. Shen, Z. Zhu, X.S. Luo, Y.S. Zhai, X.B. Hua, S.C. Zhao, L. Cen, Z.B. Zhang, A cell-free therapy for articular cartilage repair based on synergistic delivery of SDF-1 \& KGN with HA injectable scaffold, Chem. Eng. J. 393 (2020) 124649.

38. Y. Du, H. Liu, Q. Yang, S. Wang, J. Wang, J. Ma, I. Noh, A.G. Mikos, S. Zhang, Selective laser sintering scaffold with hierarchical architecture and gradient composition for osteochondral repair in rabbits, Biomaterials 137 (2017) 37-48.

39. W. Fan, L. Yuan, J. Li, Z. Wang, J. Chen, C. Guo, X. Mo, Z. Yan, Injectable double-crosslinked hydrogels with kartogenin-conjugated polyurethane nano-particles and transforming growth factor beta3 for insitu cartilage regeneration, Mat. Sci. Eng. C 110 (2020) 110705.

40. G.S. Stein, J.B. Lian, T.A. Owen, Relationship of cell growth to the regulation of tissue-specific gene expression during osteoblast differentiation, FASEB J. 4(13) (1990) 3111-3123.

41. D. Tang, R.S. Tare, L.Y. Yang, D.F. Williams, K.L. Ou, R.O. Oreffo, Biofabrication of bone tissue: approaches, challenges and translation for bone regeneration, Biomaterials 83 (2016) 363-382.

\section{Figures}




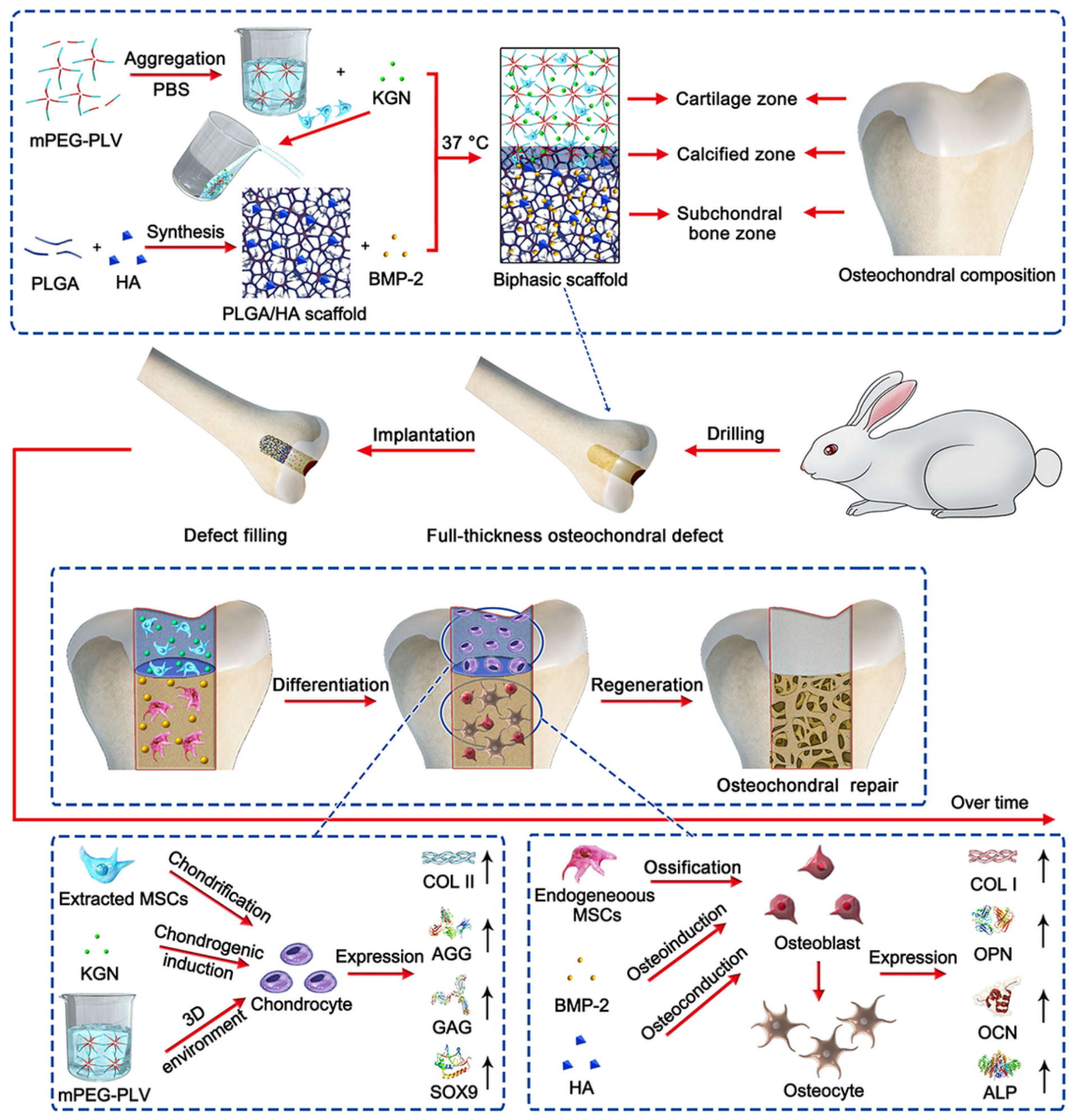

Figure 1

The process and mechanism of repairing full-thickness cartilage defect by bionic biphasic composite scaffold with osteochondrogenic factors. 
A

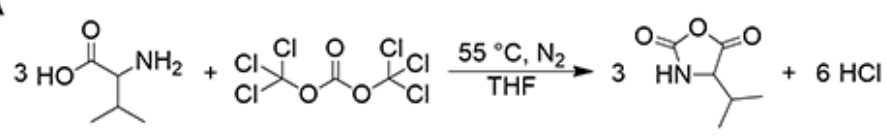

L-Val

TPG

L-Val NCA

to $\gamma_{45}^{\mathrm{NH}_{2}}+\mathrm{n} \underset{\mathrm{HN}}{ } \stackrel{3 \text { days }}{\mathrm{RT}}$

MPEG-NH

L-Val NCA

C
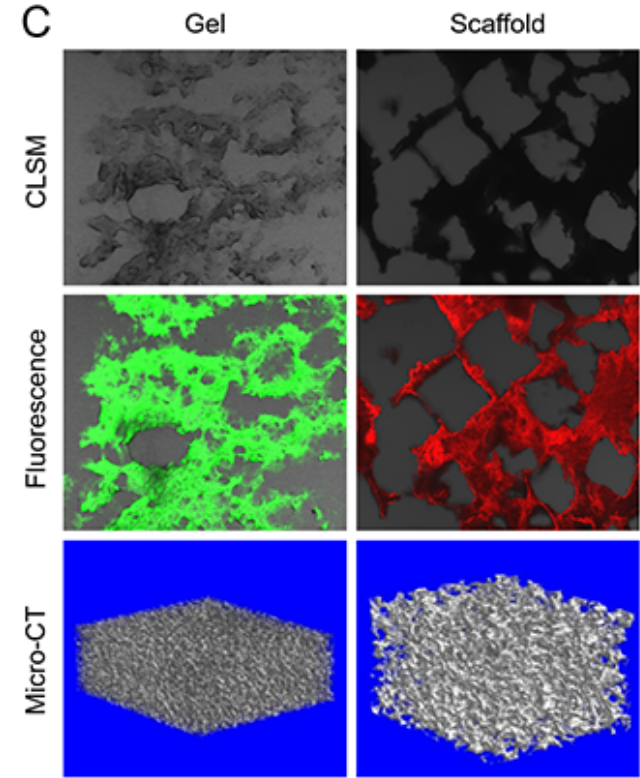

G

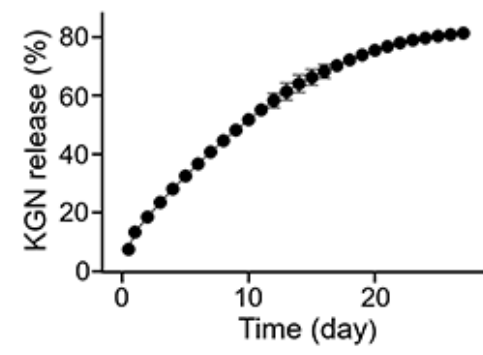

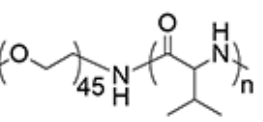

mPEG-b-PLV
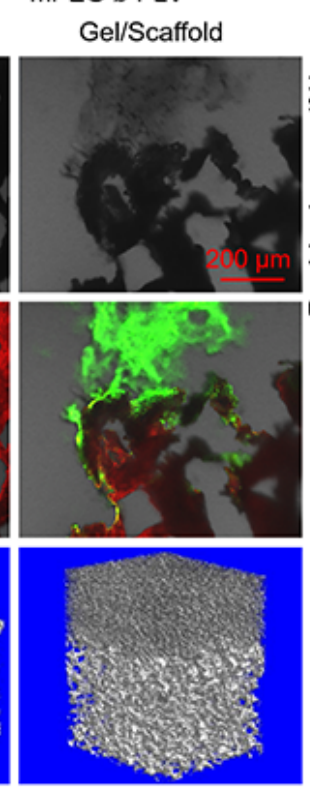

$\mathrm{H}$

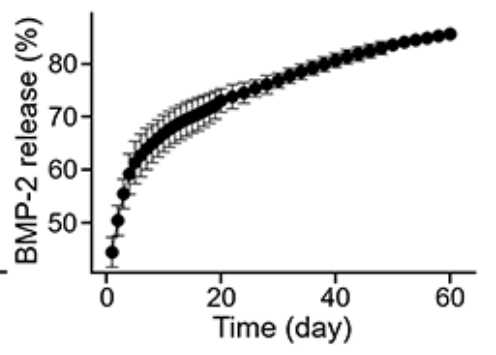

B
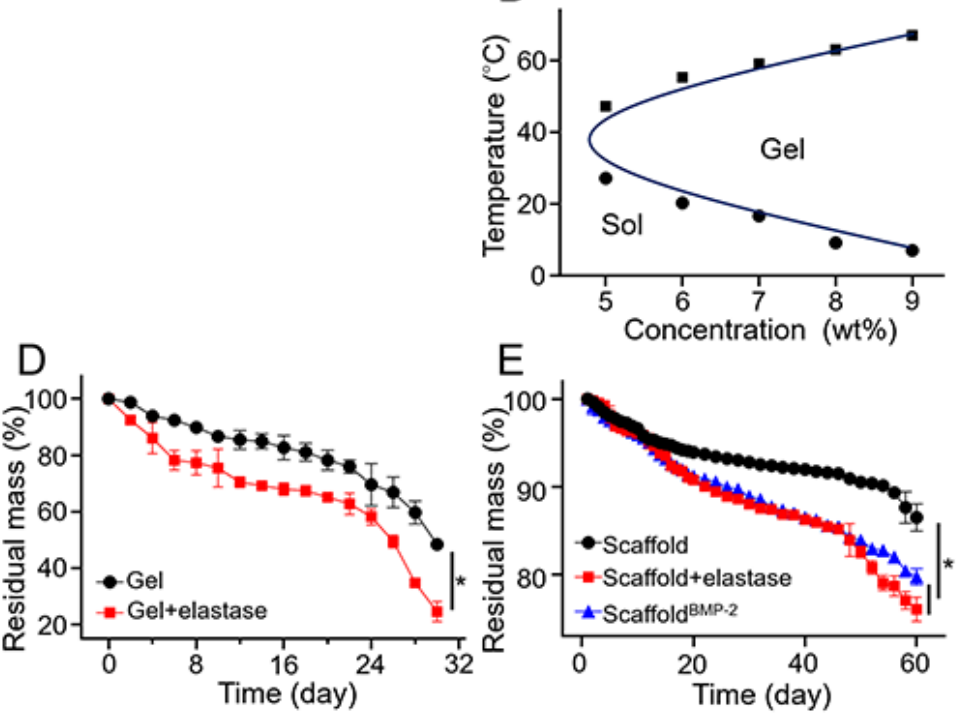

$\mathrm{E}$
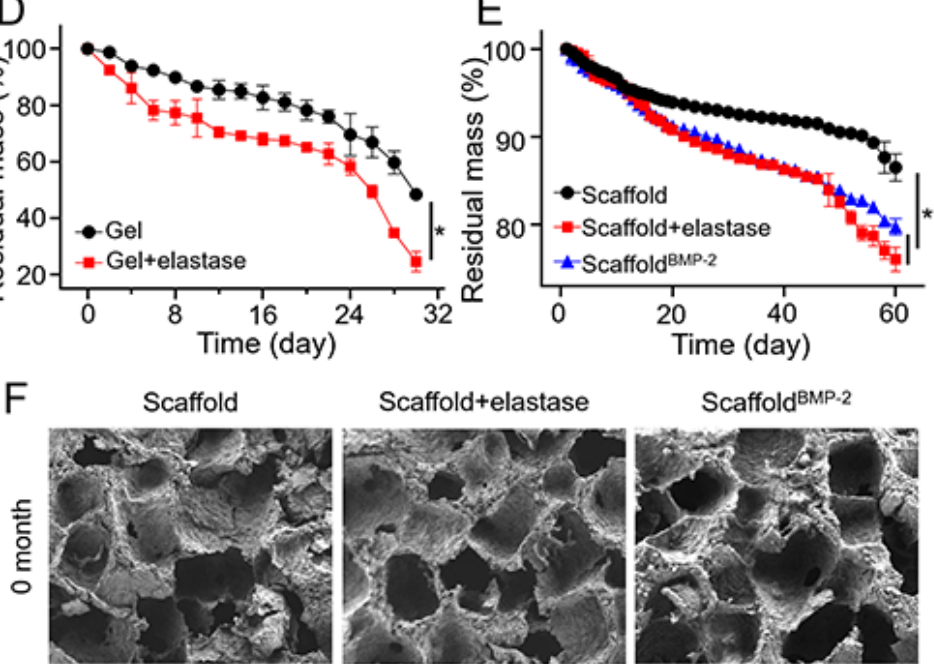

Scaffold+elastase
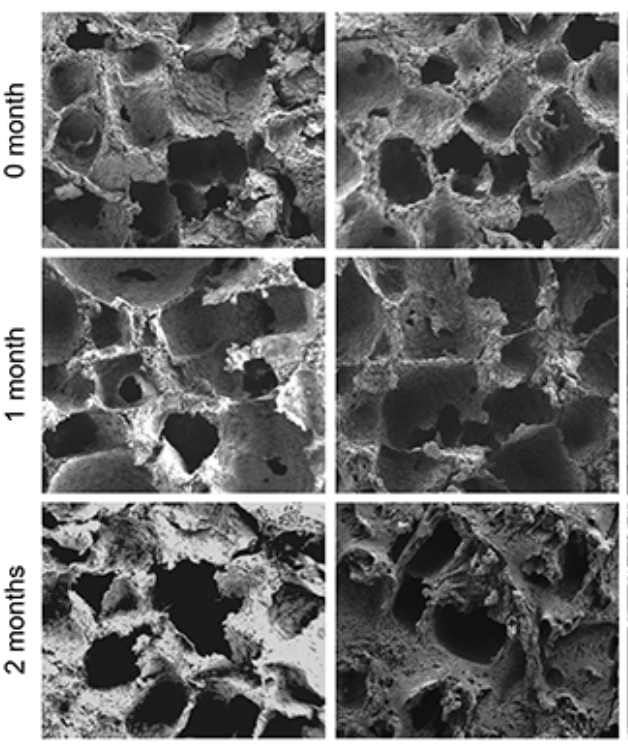

Scaffold ${ }^{B M P-2}$
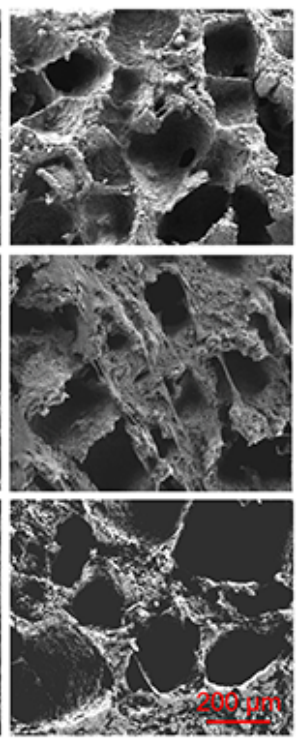

\section{Figure 2}

Synthesis and characterization of the mPEG-b-PLV thermogel and PLGA/HA scaffold. A Synthesis of the mPEG-b-PLV thermogel. B Phase diagram of the mPEG-b-PLV thermogel. C Microstructure of the mPEG-bPLV thermogel and PLGA/HA scaffold. D Residual mass of the mPEG-b-PLV thermogel ( $7 \mathrm{wt} \%)$ at $37^{\circ} \mathrm{C}$ soaked in $3.0 \mathrm{~mL}$ of Tris-HCl buffer containing $0.2 \mathrm{~g} / \mathrm{L}$ elastase with PBS as control. E Residual mass of PLGA/HA scaffold soaked in Tris-HCL buffer containing $0.5 \mathrm{~g} / \mathrm{L}$ BMP-2 or $0.2 \mathrm{~g} / \mathrm{L}$ elastase with PBS as control. F Micro-CT of the PLGA/HA scaffold with BMP-2 or elastase in in vitro degradation tests. $G$ Curves of cumulative release of KGN from the MPEG-b-PLV thermogel and H BMP-2 from the PLGA/HA scaffold. $(n=3, * P<0.05)$ 
A
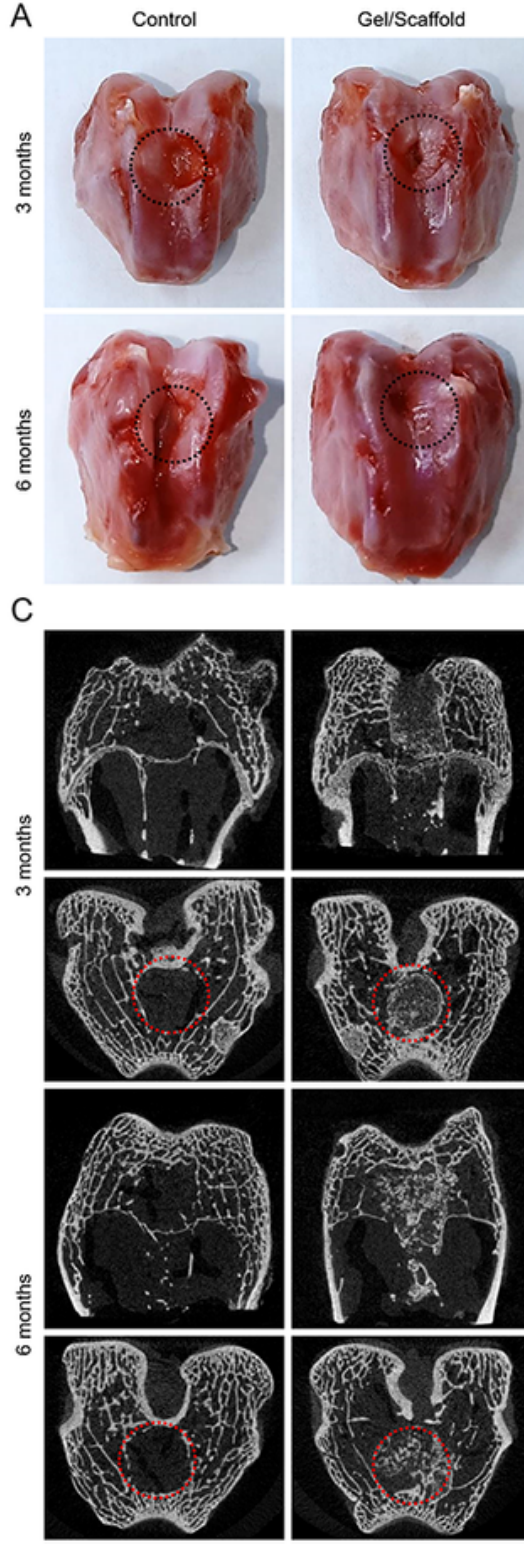

$E$
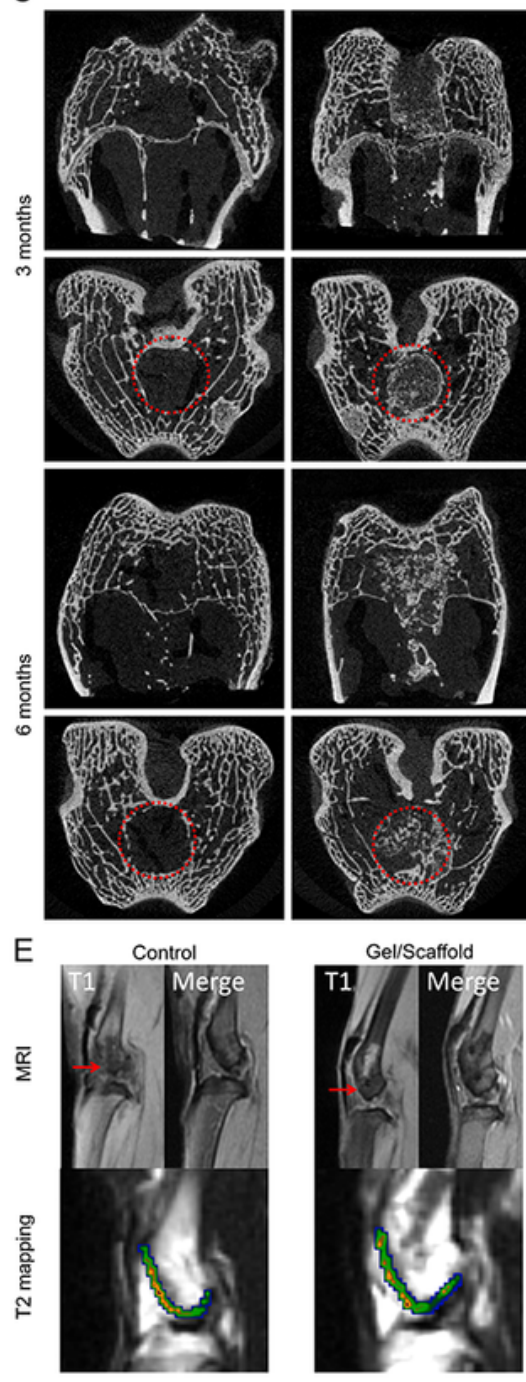

Gel-MSCs/Scaffold
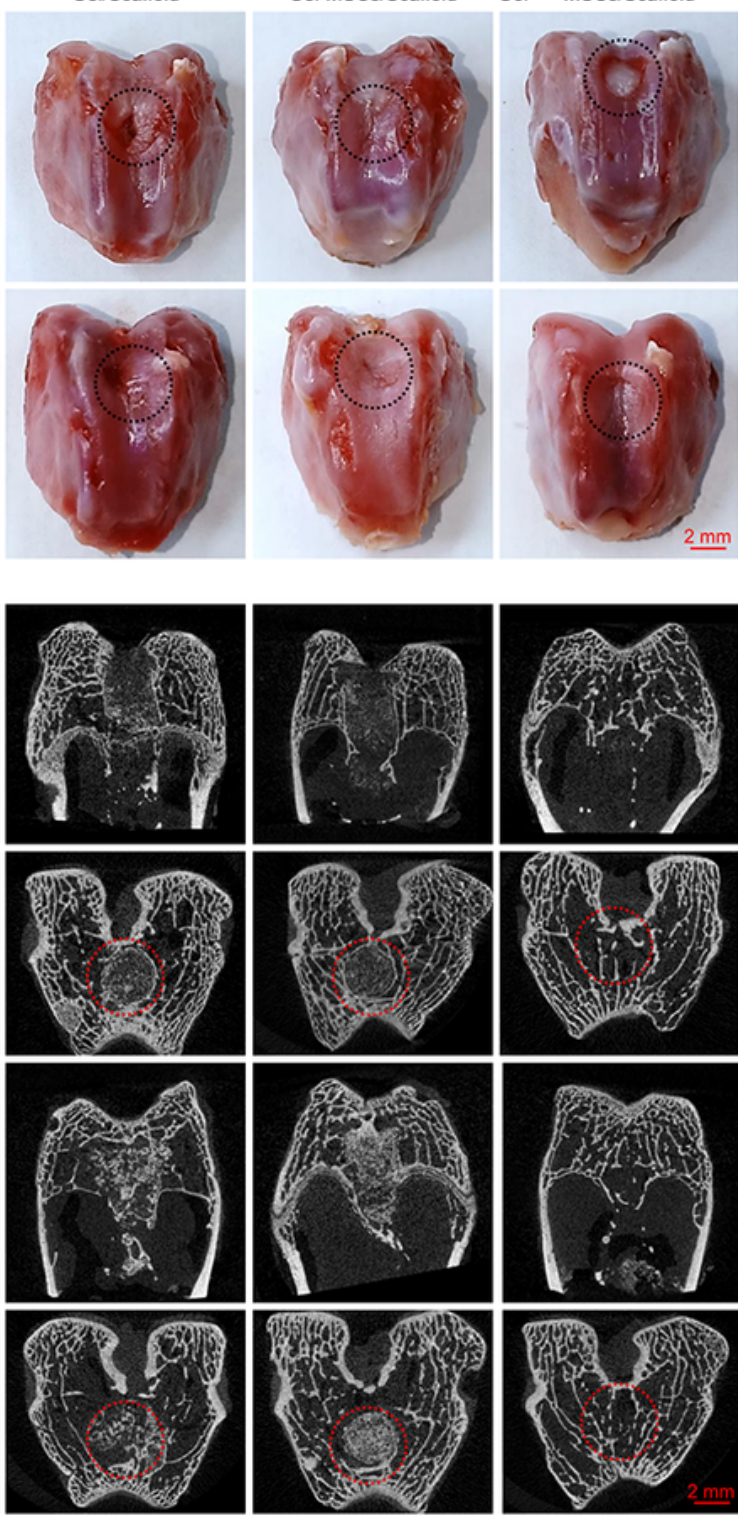

Ge|KGN-MSCs/Scaffold ${ }^{\text {MMP-2 }}$
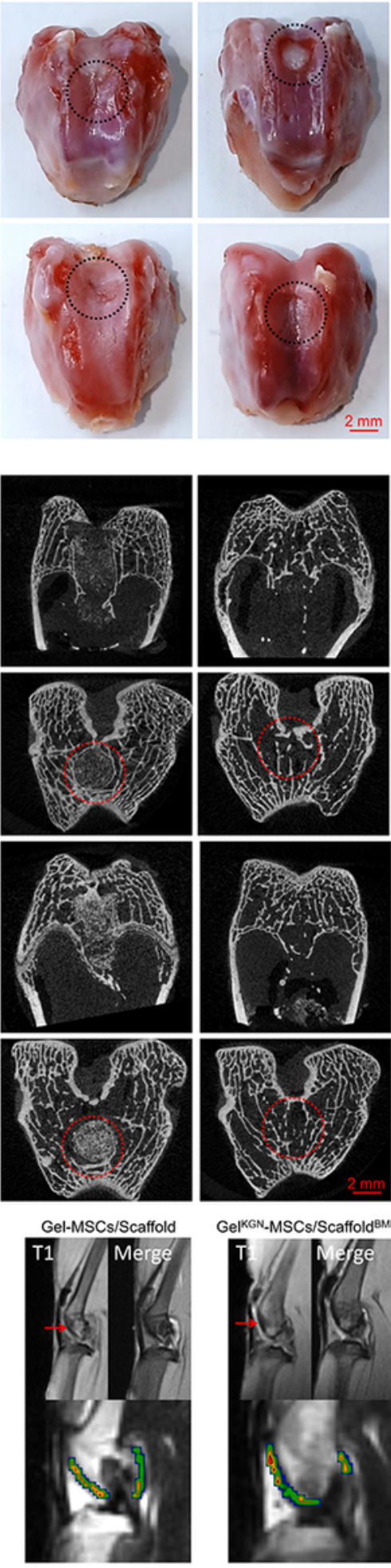

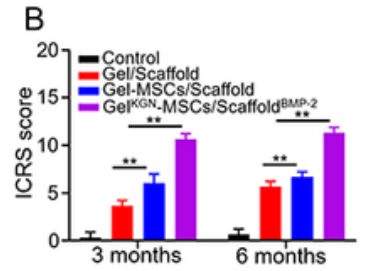

D
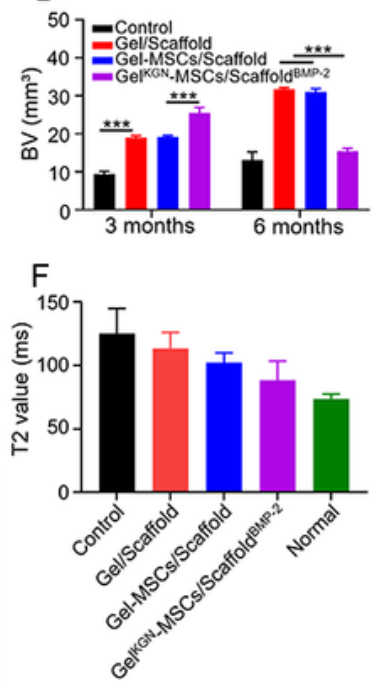

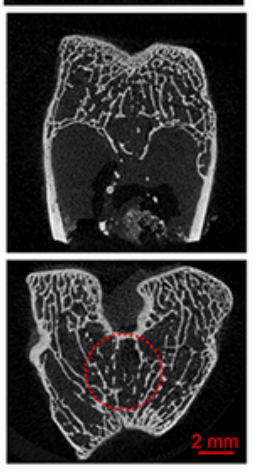

GelKGN-MSCs/Scaffold ${ }^{\text {MMP. }}$

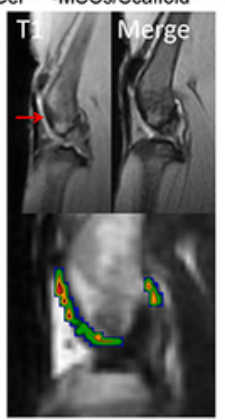

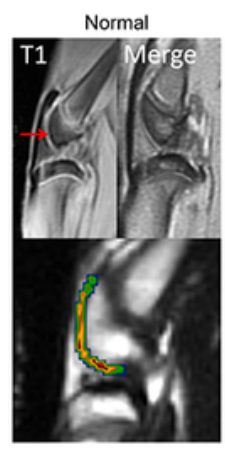

Figure 3

General manifestations and imaging examination of repaired tissues. Macroscopic view (A) and ICRS score (B) of cartilage repair at 3 and 6 months (Black circles refer to the part of osteochondral defect). Micro-CT scan (C) and BV value (D) of repaired tissues at 3 and 6 months (Red circles refer to the part of osteochondral defect). MRI imaging and T2 mapping images (E) and T2 Value (F) of the repair tissues at 6 months. All statistical data were given in the form of mean $\pm S D(n=3, * * P<0.01, * \star * P<0.001)$. 

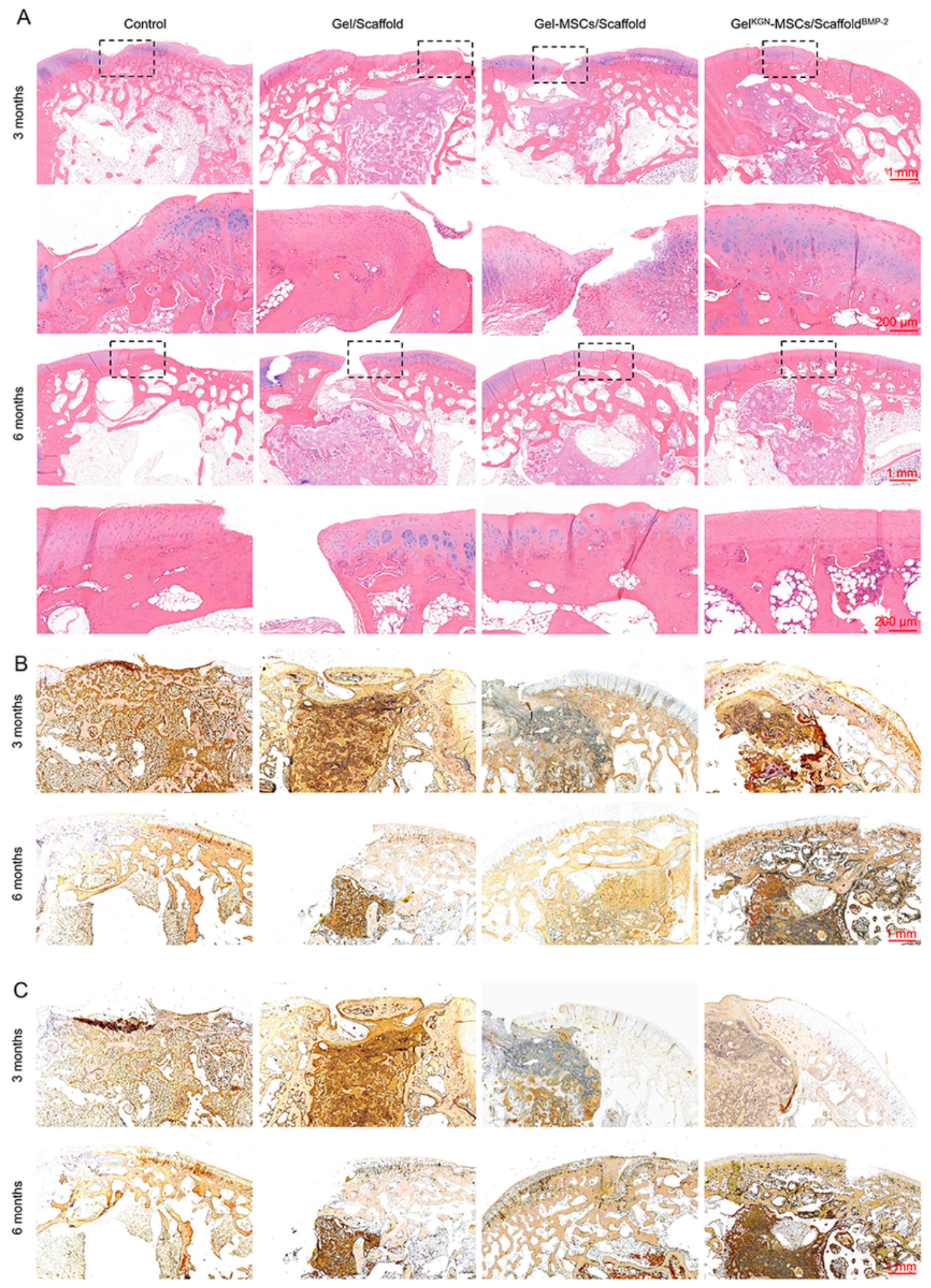

\section{Figure 4}

Histological evaluation and immunohistochemistry of the repair tissues at 3 and 6 months. H\&E staining (A), Immunohistochemistry of COL I staining (B), Immunohistochemistry of COL II staining (C). All statistical data were given in the form of mean $\pm \operatorname{SD}(n=3, \star * P<0.01)$. 

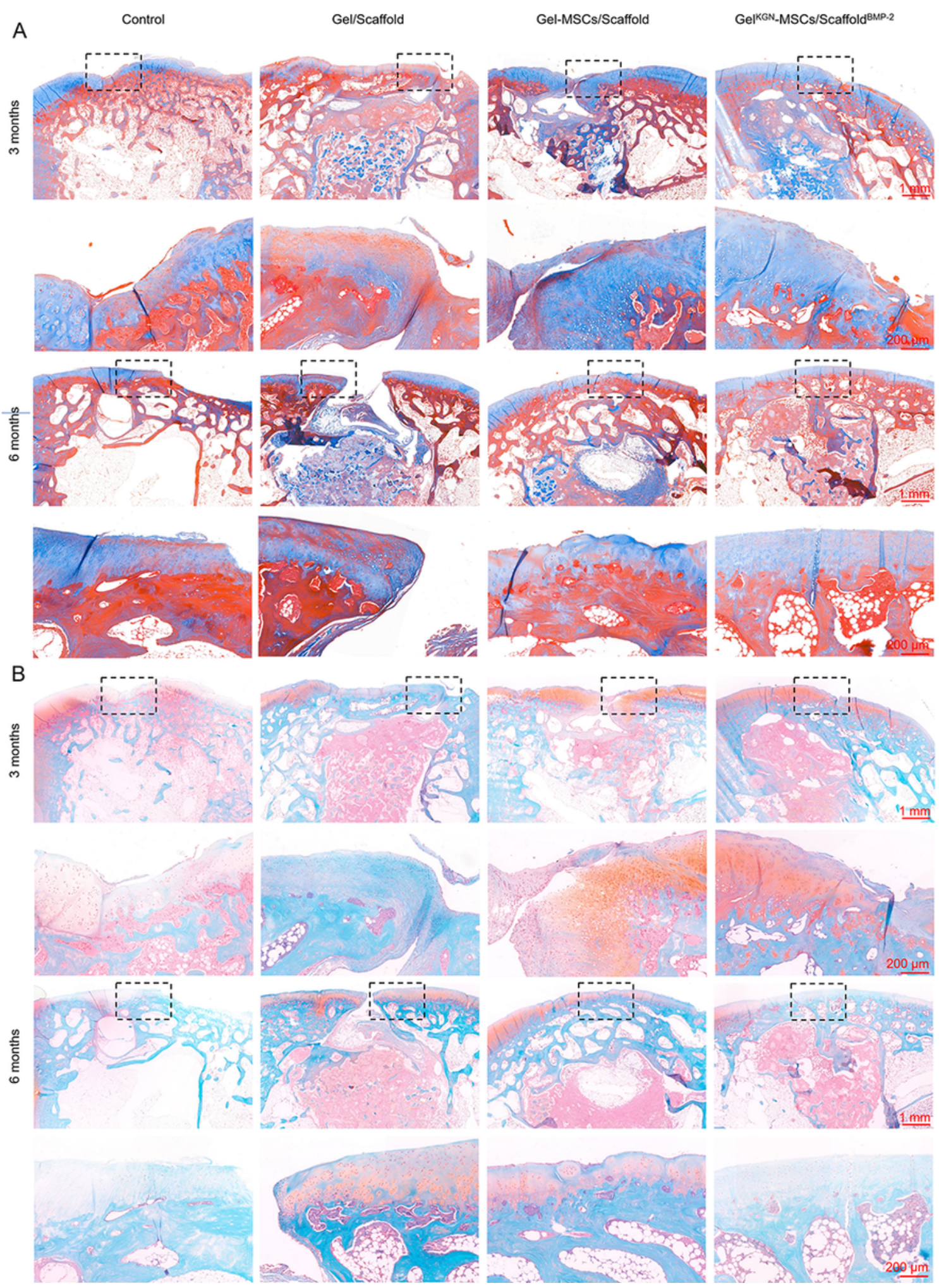

\section{Figure 5}

Masson staining (A) and Safranin O/fast green staining (B) of the repair tissues at 3 and 6 months. All statistical data were given in the form of mean $\pm S D(n=3, * \star P<0.01)$. 

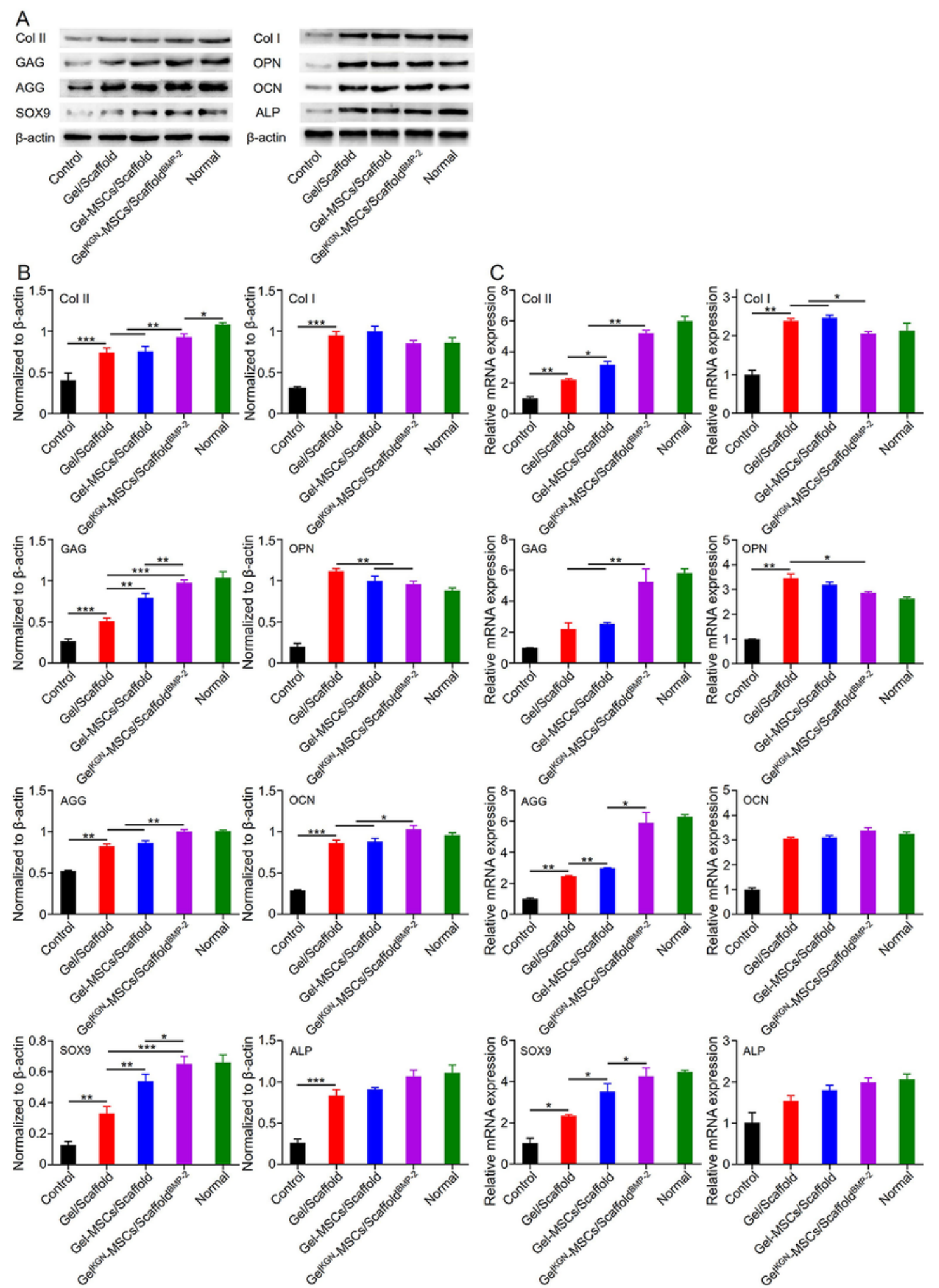

Figure 6

The expression levels of special proteins and genes in the repaired tissues analyzed by western blotting and RT-PCR 6 months post operation. A The protein expression levels of COL II, GAG, AGG, SOX9 in cartilage and COL I, OPN, OCN and ALP in subchondral bone tested by western blotting. B Semiquantitative of the western blotting. C Gene expression levels of the corresponding chondrogenic 
(COL2A1, FAM, ACAN, and SOX9) and osteoblastic (COL1A1, SPP1, SPARC, ALPL) by RT-PCR. All statistical data were given in the form of mean $\pm S D(n=3, * P<0.05, * * P<0.01, * \star \star P<0.001)$.

\section{Supplementary Files}

This is a list of supplementary files associated with this preprint. Click to download.

- graphicalabstract.docx

- Supporting20210724.doc 